\title{
INVESTIGACIONES
}

\section{PERCEPCION Y EXPECTATIVAS DE LOS ALUMNOS UNIVERSITARIOS FRENTE AL PROFESOR NO PEDAGOGO*}

\author{
Perceptions and eXPeCtations of ChILEAN University STUdents RELATED to a \\ PROFESSOR WHO ISN'T A PEDAGOGUE
}

\author{
Percepçao e expectativas dos alunos universitarios em frente \\ ao professor nao pedagogo
}
Alejandro Villalobos Clavería ${ }^{1 * *}$, Yenia Melo Hermosilla ${ }^{2}$, Cristhian Pérez Villalobos ${ }^{3}$

${ }^{1}$ Universidad de Concepción, Chile, ${ }^{2}$ Universidad de Concepción, Chile, ${ }^{3}$ Universidad del Desarrollo, Chile

\begin{abstract}
RESUMEN
El presente estudio busca aproximarse a las características de la educación superior, desde la perspectiva de los alumnos, eligiendo como foco la percepción y las expectativas que éstos tienen sobre la docencia que reciben. Para esto se encuestó a 252 alumnos, incluyendo estudiantes de pre y posgrado, aplicándoles encuestas para evaluar sus percepciones y expectativas en torno a la docencia universitaria. Los resultados muestran percepciones más positivas y expectativas más altas en el caso de los alumnos de posgrado. Las razones a la base de estas diferencias, si bien plausibles, dan luces de un cuestionamiento profundo al currículum de pregrado.
\end{abstract}

Palabras clave: profesor universitario, pedagogía universitaria, educación superior.

\begin{abstract}
This study aims at describing the characteristics of the higher education by analyzing the perceptions and expectancies of Chilean university students about the teachings they receive. For this purpose, 252 students were surveyed, including undergraduate and graduate students, applying questionnaires to evaluate their perceptions and expectations regarding higher education teachings. The results show more positive perceptions and higher expectations in the case of the graduate students. The reasons for these differences between the two groups, even though being acceptable, lead to a deeper questioning about the undergraduate curriculum.
\end{abstract}

Key words: higher education professor, university pedagogy, higher education.

\section{RESUMO}

Neste trabalho procura-se conhecer as características da educação superior, desde a visão dos alunos, escolhendo como eixo a percepção e as expectativas que os estudantes têm sobre a docência dada. 252 estudantes foram consultados, incluindo estudantes de graduação e de pós-graduação, aplicando-lhes questionários para avaliar suas percepções e expectativas em torno da docência universitária. Os resultados mostram percepções mais positivas e expectativas mais altas no caso dos estudantes de pós-graduação. As razões prováveis que justificam estas diferenças dão sinais de um questionamento profundo com respeito ao currículo de graduação.

Palavras-chave: professor universitário, pedagogía universitária, ensino superior.

* $\quad$ Estudio financiado por el proyecto FONDECYT 1070466: "La construcción del sujeto docente universitario en universidades regionales. Factores claves en su desempeño académico y profesional".

** Alejandro Villalobos Clavería. Profesor asociado. Facultad de Psicología de la Universidad de Concepción. Víctor Lamas 1290, Casilla 160-C, Concepción. Dirección de correo electrónico: avillalo@ udec.cl. Fono: 5641-2203759. 


\section{INTRODUCCION}

En una sociedad como la nuestra, marcada por el poder del conocimiento y la necesidad de adaptación en un mundo global, la educación superior constituye el motor necesario para lograr el desarrollo humano, social y cultural de los pueblos, así como su progreso científico y tecnológico (Ruiz \& Pachano, 2005).

En Chile la sociedad valora ampliamente a la educación superior, lo que se ve reflejado en la alta demanda por sus servicios de formación profesional, investigación y asistencia técnica, y en el frecuente debate público en relación al tema, que ha derivado en esfuerzos gubernamentales para regular su calidad y mejorarla (Mendes, Campbell \& de Sousa, 1990; Espinoza, 2005). La combinación de ambos factores -demanda e interés público- han favorecido un rápido crecimiento y una modificación sustantiva en el sistema de educación terciaria nacional, cuyo resultado final ha sido evaluado internacionalmente como un éxito en cambiar un sistema de élite a uno masivo, manteniendo la calidad de la educación (Organización de Cooperación y Desarrollo Económicos, OCDE, 2009), pese a los reparos internos que dicha opinión merezca.

Afirmaciones globales, como las del OCDE, deben ser entendidas en el contexto del desarrollo de educación superior chilena que ha procurado mejorar y aumentar el acceso a los diversos segmentos sociales, su preocupación por la calidad y la equidad, como también, establecer mecanismos de mejoramiento y aseguramiento de la calidad (Méndez et al., 1990; Espinoza, 2005).

En este contexto, cabe destacar que la actual política nacional de educación superior se centra en operacionalizar la fórmula de lograr un crecimiento con calidad y equidad para la sociedad chilena, dentro de una economía de mercado. En este sentido, existen desafíos y obstáculos que dificultan su expansión y la internacionalización de su quehacer.

$\mathrm{Si}$ bien en términos generales pudiésemos atribuir estas dificultades a una lógica de los sistemas de educación latinoamericanos que parece más dirigida a satisfacer las demandas empresariales que las necesidades sociales (Llomavatte, Pereyra \& Kantarovich, 2008), en lo específico, encontramos que las dificultades de la educación superior se encuentran asociadas a una variedad de factores como la creciente heterogeneidad y la falta de preparación previa de alumno usuario, la rápida expansión de la oferta de pre y posgrado, la calidad irregular de los programas de formación profesional (Espinoza, 2005), la deficiente articulación de la educación media y de la educación superior, los enfoques curriculares y didácticos en la enseñanza universitaria y el rol de las universidades en la formación del capital humano, entre otros.

Al respecto, Espinoza, Fecci, González, Marianov, Mora, Ocaranza, Prieto y Rodríguez (2006) sostienen que en Chile no ha existido un debate suficientemente riguroso sobre cómo y qué se debe enseñar en el nivel terciario, lo que ha impedido que -pese a la vigencia que tiene el tema luego del acuerdo de Bolonia- se cuente con definiciones claras de la estructura curricular que se espera para la universidad.

De esta forma, podemos observar que en nuestro país existe un modelo que busca proteger los títulos más prestigiosos socialmente, exigiendo por ley una licenciatura para éstos. Sin embargo, este modelo no se condice con las innovaciones más recientes en zonas que están liderando los cambios en la educación terciaria, como Europa y 
Estados Unidos. En el modelo europeo, por ejemplo, se está cambiando a un esquema de bachillerato de formación general y una maestría de especialización profesional posterior. En el caso norteamericano, a través de la articulación de Community Colleges, Colleges, Proffesional Schools y Graduate Schools se ha permitido el ingreso de jóvenes menos preparados generando una secuencia de especialización progresiva (Espinoza et al., 2006).

Otro elemento que Espinoza et al. (2006) destacan es la necesidad de modificar el proceso de educación continua y la duración de las carreras, reestructurando las mallas y promoviendo la incorporación de los egresados en programas de postítulo para el reciclaje y actualización. En otras palabras, se cuestiona la excesiva duración de los estudios de pregrado y la sobreespecialización; también, se plantea que un estudiante demora demasiado en obtener su primer grado. La sumatoria de estos factores incide en la alta deserción que existe a nivel de educación en Chile. Por eso, se recomienda ir a un sistema más articulado de educación superior, en el que los jóvenes puedan moverse entre los establecimientos y obtengan su primer grado después de unos tres años de estudio, sin perjuicio de los grados adicionales que deseen alcanzar posteriormente.

En este contexto, este estudio pretende ofrecer otros resultados que pueden ayudar a mejorar la comprensión del funcionamiento interno universitario, según la mirada de sus principales usuarios: los estudiantes. Específicamente, este estudio se centrará en la perspectiva que estos actores tienen de la docencia universitaria, nivel ejecutor a través del cual se expresan los lineamientos, capacidad de gestión, recursos y potencialidades de las casas de estudio superiores, ya que son éstos finalmente quienes deben llevar a la práctica las innovaciones y mejoras del sistema (Solar \& Díaz, 2007).

Las universidades chilenas tienen como función principal formar capital humano de avanzada. Por tanto, la docencia y la formación profesional son ejes centrales de su quehacer institucional; responsabilidad ejercida por profesionales y especialistas que, en muchas ocasiones, no tiene una formación pedagógica para responder a las nuevas exigencias que supone la modernización de la universidad. Debido a esto, el presente estudio ha decidido focalizarse en el caso de la docencia de quienes no son profesores de origen. Esto, pues, pese a las características de la docencia universitaria, la mayoría de los académicos -médicos, abogados, ingenieros, psicólogos, etc.- no han recibido formación pedagógica e incluso la perciben como algo ajeno a sus disciplinas (Escudero, 2003).

A partir de lo anterior, el presente estudio proyecto FONDECYT 1070466: "La construcción del sujeto docente universitario en universidades regionales. Factores claves en su desempeño académico y profesional", tiene por objetivo analizar percepciones y expectativas que tienen de sus profesores no pedagogos. Para esto se centra en dos universidades regionales, ubicadas al sur de Santiago, capital de Chile. Estas universidades, una estatal y la otra comunitaria, son consideradas como eje del desarrollo local y ambas acreditadas por la Comisión Nacional de Acreditación (CNA).

\section{METODO}

El estudio se realizó a través de un diseño no experimental transversal con un alcance correlacional. 


\section{PARTICIPANTES}

La población estuvo compuesta por alumnos de pre y posgrado de dos universidades tradicionales chilenas de la zona sur del país, específicamente de las ciudades de Concepción y Osorno. Se eligió una muestra de 252 estudiantes, a través de un muestreo no probabilístico por accesibilidad, logrando una representación cercana al 50\% de las dos casas de estudio (51,6 y 48,4\%, respectivamente). Se cubrieron 17 carreras de pregrado, 10 de magíster y 3 de doctorado de diferentes áreas del conocimiento, con un 78,2\% de alumnos de pregrado $(n=197)$ y un $18,7 \%$ de posgrado $(n=47)$.

Las edades de los participantes se ubicaron entre 19 y 50 años, con una media de 23,66 años $(D . E .=4,23)$. Un 55,2\% $(n=139)$ de los participantes era hombre y un $44,0 \%(n=111)$ mujer, con dos casos $(0,8 \%)$ que no entregaron dicha información.

\section{INSTRUMENTOS}

Se aplicó a los estudiantes una batería de dos instrumentos

a) Escala de Percepción de la Docencia Universitaria (EPDU): Validado en población universitaria por Villalobos, Melo y Pérez (en revisión, a), esta escala evalúa la percepción que los alumnos tienen de sus docentes considerando una escala global y tres subescalas: Calidad del docente, calidad de la enseñanza y organización del entorno educativo. Se compone de 18 ítems en formato Likert, en el que se presentan distintas descripciones del docente y su actividad, solicitándole al sujeto que elija la alternativa de respuesta que más se ajuste a su opinión sobre un docente en particular. Para esto puede utilizar una de cinco alternativas $(1=$ Completamente en desacuerdo; 2 = En desacuerdo; 3 = Ni de acuerdo ni en desacuerdo; 4 = De acuerdo, y 5 = Completamente de acuerdo). En el presente estudio la escala general obtuvo una confiabilidad, según el coeficiente alfa de Cronbach de 0,91; las subescalas, por su parte, presentaron confiabilidades de 0,86, 0,78 y 0,70, respectivamente.

b) Escala de Expectativas asociadas a la Docencia Universitaria (EADU): Elaborado y validado por los investigadores en población universitaria (Villalobos, Melo \& Pérez, en revisión, b), evalúa las expectativas que los alumnos tienen sobre dos dimensiones de las actividades docentes en las que van a participar: Adecuación del proceso educativo y Beneficios de la participación en el proceso educativo. Se compone de 15 ítems en formato Likert, en el que se presentan diversas consecuencias potenciales de la docencia, ante las que el sujeto debe responder eligiendo, entre cinco posibilidades, la alternativa de respuesta que más se ajuste a su opinión ( 1 = Completamente en desacuerdo; 2 = En desacuerdo; 3 = Ni de acuerdo ni en desacuerdo; 4 = De acuerdo, y 5 = Completamente de acuerdo). En el estudio, las subescalas presentaron confiabilidades, según el coeficiente Alfa de Cronbach, de 0,85 y 0,74. Adicionalmente, la escala general presentó una confiabilidad de 0,88 .

c) Cuestionario sociodemográfico: Finalmente, este instrumento consultaba por el sexo del participante, su edad, nivel de estudios, programa que cursaba, facultad, universidad y estado civil. 


\section{PROCEDIMIENTO}

Se hizo una selección de académicos interesados en temas de docencia universitaria, los cuales fueron invitados a participar en las diferentes etapas de proyecto, mediante una carta de convocatoria. Las respuestas afirmativas fueron agrupadas por disciplina, experiencia docente y tiempo disponible para establecer un grupo heterogéneo de académicos. Posteriormente, se les contactó para aplicar la encuesta en uno de sus cursos a cargo, en donde se pidió a los alumnos que respondieran teniendo en mente al docente participante.

Previo a responder, se les solicitó a los alumnos firmar un consentimiento informado en donde se describía el propósito de la investigación en la que participaban y las características de su participación (anómina, confidencial y voluntaria).

\section{ANALISIS DE LOS DATOS}

Para el análisis de los datos se utilizó estadística descriptiva y, para someter a prueba las hipótesis, se empleó la prueba paramétrica $t$ de Student en base a un contraste unilateral.

\section{RESULTADOS}

El análisis de los datos se orientó a partir de las siguientes hipótesis de investigación:

1. Existe diferencias en la percepción que los alumnos de pregrado y posgrado tienen de sus docentes.

2. Existen diferencias en las expectativas que los alumnos de pregrado y posgrado tienen de la docencia universitaria.

3. La percepción que hombres y mujeres tienen de sus docentes es diferente.

4. Las expectativas que hombres y mujeres tienen de la docencia es diferente.

Antes de someter a prueba estas hipótesis, se obtuvo el puntaje de cada una de la escala general del EPDU y sus tres subescalas, y de la escala general del EADU y sus dos subescalas. Para esto se calculó la media aritmética de todos los ítems que constituían cada escala, obteniendo un mismo rango posible de variación para todas ellas de 1 a 5 , lo que facilita su comparación.

En la Tabla 1 se presenta un resumen de la media muestral por cada escala y subescala, además de sus correspondientes estadísticos de dispersión y distribución. Los estadísticos de asimetría y curtosis apoyan la pertinencia de utilizar una prueba paramétrica.

En relación a la primera hipótesis del estudio, utilizando la prueba $t$ de Student para muestras independientes en base a un contraste bilateral, se encontró que en todos los instrumentos los alumnos de posgrado tenían una evaluación más favorable que los alumnos de pregrado. 
Tabla 1

Estadísticos descriptivos de las escalas EPDU y EADU

\begin{tabular}{|l|c|c|c|c|c|c|c|c|}
\hline & Mín. & Máx. & M & \multicolumn{1}{c|}{ D.E. } & \multicolumn{2}{|c|}{ Asimetría } & \multicolumn{2}{|c|}{ Curtosis } \\
\hline & Estadístico & Estadístico & Estadístico & Estadístico & Estadístico & $\begin{array}{c}\text { Error } \\
\text { típico }\end{array}$ & Estadístico & $\begin{array}{r}\text { Error } \\
\text { típico }\end{array}$ \\
\hline $\begin{array}{l}\text { EPDU - Escala } \\
\text { general }\end{array}$ & 1,83 & 5,00 & 3,7818 &, 57148 &,- 218 &, 154 &, 095 &, 307 \\
\hline $\begin{array}{l}\text { EPDU - Calidad } \\
\text { del docente }\end{array}$ & 1,56 & 5,00 & 3,8635 &, 64325 &,- 486 &, 154 &, 381 &, 307 \\
\hline $\begin{array}{l}\text { EPDU - Calidad de } \\
\text { la enseñanza }\end{array}$ & 1,80 & 5,00 & 3,7758 &, 62164 &,- 169 &, 154 &, 053 &, 307 \\
\hline $\begin{array}{l}\text { EPDU - Organi- } \\
\text { zación del entorno } \\
\text { educativo }\end{array}$ & 1,00 & 5,00 & 3,6033 &, 73930 &,- 400 &, 154 &, 185 &, 307 \\
\hline $\begin{array}{l}\text { EADU - Escala } \\
\text { general }\end{array}$ & 2,13 & 5,00 & 4,0336 &, 55651 &,- 584 &, 154 &, 249 &, 307 \\
\hline $\begin{array}{l}\text { EADU - Adecua- } \\
\text { ción del proceso } \\
\text { educativo }\end{array}$ & 2,00 & 5,00 & 3,9605 &, 59865 &,- 539 &, 154 &, 112 &, 307 \\
\hline $\begin{array}{l}\text { EADU - Beneficios } \\
\text { de la participación } \\
\text { en el proceso } \\
\text { educativo }\end{array}$ & 2,00 & 5,00 & 4,1792 &, 63364 &,- 842 &, 154 &, 377 &, 307 \\
\hline
\end{tabular}

En primer lugar, considerando la escala general del EPDU, la percepción que los alumnos de posgrado $(M=4,17 ; D . E .=, 52)$ tenían de la docencia que recibían era más favorable que la de los alumnos de pregrado $(M=3,72 ; D . E .=4,17), \mathrm{t}_{(240)}=-5,37$; $\mathrm{p}<0,001$. Esto también ocurría en el caso de las subescalas de Calidad del docente $\left(\mathrm{t}_{(240)}=-5,64 ; \mathrm{p}<0,001\right)$, Calidad de la enseñanza $\left(\mathrm{t}_{(240)}=-3,18 ; \mathrm{p}<0,01\right)$ y Organización del entorno educativo $\left(\mathrm{t}_{(240)}=-3,79 ; \mathrm{p}<0,001\right)$.

En relación a las expectativas se encontró que los alumnos de posgrado tenían a su vez expectativas más positivas sobre la adecuación del proceso educativo $(M=4,22$; $D . E .=, 57)$ y sobre los beneficios de la participación en éste $(M=4,49 ; D . E .=, 45)$, que los alumnos de pregrado $(M=3,89 ; D . E .=, 58$ y $M=4,10 ; D . E .=, 64$, respectivamente), con resultados de $\mathrm{t}_{(240)}=-3,51 ; \mathrm{p}<0,01$ para la primera escala $\mathrm{y} \mathrm{t}_{(96,11)}=-4,84$; $\mathrm{p}<0,001$ para la segunda. Cabe mencionar que en este último caso se consideraron varianzas diferentes. En relación a la escala general del EADU, también los alumnos de posgrado asociaron expectativas más positivas a la docencia $(M=4,31 ; D \cdot E .=, 48)$ que el grupo de pregrado $(M=3,96 ; D . E .=, 54), \mathrm{t}_{(240)}=-4,04 ; \mathrm{p}<0,001$.

Dadas las diferencias encontradas entre pregrado y posgrado, se decidió realizar la comparación por género diferenciando ambos niveles.

A nivel de pregrado, el estadístico $t$ de Student para muestras independientes, asumiendo varianzas iguales, no arrojó diferencias asociadas al sexo entre la percepciones de la docencia universitaria, ni a nivel de la escala general de la EPDU $\left(\mathrm{t}_{(191)}=-3,45\right.$; $\mathrm{p}=0,731)$, ni en relación a la percepción de la calidad del docente $\left(\mathrm{t}_{(191)}=-, 232\right.$; 
$\mathrm{p}=, 817)$, la calidad de la enseñanza $\left(\mathrm{t}_{(191)}=-, 754 ; \mathrm{p}=, 452\right)$ o la organización del entorno educativo $\left(\mathrm{t}_{(242,96)}=-, 014 ; \mathrm{p}>0,989\right)$.

Sin embargo, a nivel de expectativas sí se encontraron diferencias significativas. Con el mismo estadístico, asumiendo varianza diferentes, se encontró que las mujeres $(M=4,11 ; D \cdot E .=0,40)$ presentaban expectativas más elevadas que los hombres $(M=3,83$; $D . E .=0,61)$ en torno a la docencia en general, $\mathrm{t}_{(180,434)}=-3,82 ; \mathrm{p}<0,001$.

Lo mismo ocurría en el caso de las expectivas en torno a la adecuación del proceso educativo, en donde las mujeres presentaban una media de 4,00 (D.E. $=, 47)$ en comparación con la media de $3,79(D . E .=, 64)$ presentada por los hombres, $\mathrm{t}_{(188,078)}=-2,62$; $\mathrm{p}<0,05$, y en el caso de las expectativas sobre los beneficios de la propia participación en el proceso educativo, en donde las mujeres $(M=4,32 ; D . E .=0,47)$ nuevamente reportaron expectativas mayores que los hombres $(M=3,90 ; D . E .=0,70), \mathrm{t}_{(242,96)}=-3,69$; $\mathrm{p}>0,001$.

A nivel de posgrado, si bien ocurre el caso contrario, con los hombres presentando puntuaciones en casi todas las escalas de percepciones levemente superiores a las de las mujeres, éstas no son estadísticamente significativas ni a nivel de la escala general del EPDU $\left(\mathrm{t}_{(45)}=, 63 ; \mathrm{p}=, 53\right)$, ni en sus subescalas de calidad docente $\left(\mathrm{t}_{(45)}=, 68 ; \mathrm{p}=, 50\right)$ $\mathrm{y}$ organización del entorno educativo $\left(\mathrm{t}_{(45)}=1,07 ; \mathrm{p}=, 29\right)$. En el caso de la escala de calidad de la enseñanza, que es la única en que las mujeres $(M=4,04 ; D . E .=0,62)$ presentan una media muestral mayor a la de los hombres $(M=4,00 ; D . E .=0,56)$, tampoco hay diferencias significativas $\left(\mathrm{t}_{(45)}=-, 20 ; \mathrm{p}=, 84\right)$. Lo mismo ocurre en la EADU, donde las expectativas levemente superiores de las mujeres sobre los hombres no alcanzan significación estadística ni a nivel de la escala general $\left(\mathrm{t}_{(45)}=, 25 ; \mathrm{p}=, 80\right)$ ni a nivel de las subescalas de adecuación del proceso educativo $\left(\mathrm{t}_{(45)}=, 30 ; \mathrm{p}=, 77\right) \mathrm{y}$ beneficios asociados a la participación en éste $\left(\mathrm{t}_{(45)}=, 05 ; \mathrm{p}=, 96\right)$.

\section{CONCLUSIONES}

La globalización como paradigma de la vida contemporánea impone al mundo académico la necesidad de adaptarse a este proceso de cambio global y la renovación académica y curricular aparece como un imperativo.

En este sentido, conocer las percepciones y expectativas que tienen los estudiantes universitarios sobre la docencia universitaria ayuda a promover un cambio en las estructuras y prácticas pedagógicas de la enseñanza superior. Los datos obtenidos revelan la necesidad de revisar la actual concepción que se tiene de la docencia universitaria, sobre todo a nivel de la educación de pregrado en donde los alumnos reportan una percepción menos positiva de la docencia y, lo que requiere de mayor atención, menores expectativas.

En este sentido, parece lícito plantearse que las mayores expectativas y la percepción más positiva que presentan los alumnos de posgrado se deban a que éste se encuentra en una situación diferente al haber elegido un programa teniendo mayor conocimiento sobre las características de éste y la forma en que puede beneficiarle en su profesión; una situación diferente a la que enfrenta la mayoría de los egresados de enseñanza media al elegir una carrera de pregrado. Lo anterior permitiría que en un magíster, un doctorado o incluso otros programas como postítulos o diplomados, el estudiante tenga expectativas no sólo mayores sino más definidas en relación a la docencia, lo que deviene en mayor 
motivación por involucrarse en el proceso, lo que a su vez le permitiría sacar -por sus propios medios- un mayor provecho de la actividad del docente.

También podemos considerar que en posgrado, pese a la gran heterogeneidad de la oferta en nuestro país, en general se tiene un proceso de selección más exigente para los docentes que imparten clases en él, lo que puede hacer que: por un lado, él tenga un mayor dominio conceptual y práctico de los contenidos que imparte, haya desarrollado mejores estrategias para entregar contenidos de alto nivel de especialización y tenga un manejo más acabado de los recursos que puede emplear para ello, lo que haría que el alumno lo percibiera de mejor forma. Por otro lado, un estudiante puede esperar estas mismas características en posgrado, aumentando sus expectativas, lo que se acentúa al considerar que aún en nuestra sociedad se le entiende como un nivel electivo, donde la voluntariedad de la participación y los costos usualmente asociados (por ejemplo, para adultos que inician este tipo de estudios y tienen que combinarlos con su trabajo y sus familias) podrían aumentar el nivel de exigencia del estudiante.

Pese a estas posibles explicaciones, que hacen comprensible las diferencias encontradas, es necesario plantearse de qué forma pueden ser indicadores de la inadecuación del sistema. Si asumimos que un alumno de enseñanza media puede tener expectativas menos definidas de qué puede esperar de una carrera universitaria, pero aún así debe ingresar a una, ¿por qué lo mantenemos durante cuatro o cinco años en un programa que no pudo elegir con toda la información necesaria? Si la voluntariedad de la participación y la posibilidad de elegir pueden aumentar la motivación de las personas, ¿por qué le negamos al alumno la posibilidad de seguir eligiendo voluntariamente su camino de formación profesional? Si asumimos que el mundo globalizado, hiperconectado y competitivo está en constante cambio y que los campos de ejercicio laboral se deben adaptar a él, ¿por qué mantenemos al alumno en sistema de formación durante largos periodos de tiempo que rigidizan su posibilidad de adaptación?

En este sentido, los resultados de este estudio buscan abrir el cuestionamiento, no de las razones que hacen que los alumnos de posgrado evalúen mejor y le exijan más a sus procesos educativos, ya que éstas son identificables con relativa facilidad. Lo que parece más valioso aún, es cuestionarnos de qué forma el que aparezcan estas razones como realidades de la educación terciaria pueden mostrar riesgos severos que atentan contra su calidad y contra su capacidad de cumplir con los objetivos que nosotros como sociedad le hemos impuesto.

\section{BIBLIOGRAFIA}

Espinoza, O. (2005). Privatización y comercialización de la educación superior en Chile: Una visión crítica. Revista de Educación Superior, 135 (3), 41-60.

Espinoza, O., Fecci, E., González, L. Marianov, V., Mora, A., Ocaranza, O, Prieto, J. y Rodríguez, E. (2006) Informe: Educación Superior en Iberoamérica El Caso de Chile. Santiago: Centro Interuniversitario de Desarrollo, CINDA.

Llomavatte, S., Pereyra, K. y Kantarovich, G. (2008). Escribir sobre el vínculo universidad-sociedad. Llamar a las cosas por su nombre es complejo. Revista del Instituto de Investigaciones en Ciencias de la Educación, 26, 37-55.

Mendes, A., Campbell, J. C. y de Sousa, R. (2005). La educación superior en Chile: continuidades y desafíos. Fundamentos en humanidades, 6 (11), 9-20. 
Organización para la Cooperación y el Desarrollo Económicos, OCDE (2009). La educación superior en Chile. París, Francia.

Solar, M. I. y Díaz, C. (2007). El sistema de cogniciones y creencias del docente universitario y su influencia en su actuación pedagógica. Horizontes educacionales, 12 (1), 35-42.

Ruiz, L. y Pachano, L. (2005). La docencia universitaria y las prácticas evaluativas. La revista venezolana de educación, 9 (31), 531-540.

Villalobos, A., Melo, Y. y Pérez, C. (en revisión, a). Propiedades psicométricas de la Escala de Percepción de la Docencia Universitaria en estudiantes chilenos. Revista de estudios sobre educación.

Villalobos, A., Melo, Y. y Pérez, C. (en revisión, b). Propiedades psicométricas de la Escala de Expectativas sobre a la Docencia Universitaria. Revista lationamericana de psicología. 
